\title{
Learning how to learn schemata
}

ED M. EDMONIDS, SELBY H. EVANS AND MARVIN R. MUELLER

TEXAS CHRISTIAN UNIVERSITY

Ss, reproducing from memory patterns which had a schema, performed better when pretrained with patterns having other schemata than when pretrained with patterns having no schema. Performance also improved within each block of patterns having the same schema. Neither type of improvement was contingent on knowledge of results.

Edmonds \& Evans (1966a) found that, in a reproduction task, Ss benefitted more from training with patterns having a common schema (transfer group) than from training with random patterns. This result indicated that the Ss in the transfer group abstracted the schema in the set of patterns (a "specific encoding principle'") and used it to remember and reproduce individual patterns. This schema learning was not contingent upon knowledge of results. In another study (Edmonds \& Evans, 1966b), the best-fitting equation describing schema learning in this previous research was found quite accurately to predict performance with samples of patterns randomly selected from four different populations. This successful prediction was attributed to a pattern generation procedure (VARGUS 7) which allowed manipulation of the parameters describing the different pattern populations.

In brief, VARGUS 7 (Evans, 1964; Evans \& Edmonds, 1966) is a computer program which allows quantification of patterns in informational terms and independent manipulation of redundancy and form of schema. The schema in patterns generated by VARGUS 7 consists of a most probable sequence (MPS) of column heights as determined by the transitional probabilities of a seven element Markov process. Redundancy is determined by the magnitude of the probabilities associated with the schematic sequences (MPS's).

The present study, an extension of those above, was designed to investigate the development of a "general encoding principle" in a reproduction task (without knowledge of results). In this context, a "general encoding principle" involves learning that sets of patterns containing different MPS's can be most effectively reproduced by discovering and using the particular MPS associated with each set of patterns. A general encoding principle, while requiring a task involving memory, rather than a discrimination task (Edmonds \& Evans, 1966a; Mueller, Edmonds, \& Evans, 1966), is analogous to Harlow's (1949) concept of learning set, in that practice in successively learning different schemata should lead to a general improvement in schema learning.

A transfer group was trained by having each $\mathrm{S}$ reproduce a sequence of $67 \%$ redundant patterns which consisted of 15 patterns containing one MPS, followed by 15 patterns containing a second MPS, followed by 15 patterns containing a third MPS. In the test phase, this group reproduced 15 patterns containing a fourth MPS. A control group reproduced $450 \%$ redundant (no schema) patterns in the training phase and had the same task as the transfer group in the test phase. The hypothesis tested was that the transference of a general encoding principle would be evidenced by the transfer group's performing significantly better than the control group in the test phase.

\section{Method}

Subjects. The Ss were 56 undergraduates enrolled in psychology courses at Texas Christian University. They were randomly assigned to the training groups.

Patterns. Each of four different MPS's, designated pattern set PS1, PS2, PS3, and PS4, was used in the VARGUS 7 program to produce $67 \%$ redundant patterns containing 12 columns. A fifth set of 12 column $0 \%$ redundant patterns (PS5) was produced by making all column height sequences equiprobable. PS5 contained 45 patterns, and the other sets contained 15 patterns each.

Task and procedure. The experiment consisted of four tasks (A, B, C, and D) presented in succession without interruption. The Ss were not told when one task ended and the next began. For the transfer group, each task consisted of 15 reproduction trials with 15 different patterns having the same schema. The transfer group $(\mathrm{N}=28$ ) was subdivided into four subgroups and the order of presentation of the four schemata was counterbalanced with a Latin square arrangement. Thus, all four schemata were equally represented in each task. For the control group $(\mathrm{N}=28)$ tasks $\mathrm{A}, \mathrm{B}$, and $\mathrm{C}$ consisted of 15 reproduction trials with 15 patterns from PS5. A new set of patterns was used for each task. For task D, the control group was subdivided into four subgroups and each subgroup was given 15 reproduction trials on a different set of redundant patterns (i.e., the control group received the same task Das did the transfer group). In all other respects, the task and procedure were identical to that described by Edmonds \& Evans (1966b), which reported results derived from task A of the present experiment. Patterns were reproduced from memory, and no knowledge of results was given.

\section{Resulfs and Discussion}

A 2 by 4 ( 2 groups by 4 MPS's) analysis of variance was used to evaluate performance differences between the transfer and control groups on the first five trials of task D. The transfer group was superior, $F=4.05$, $\mathrm{df}=1 / 48, \mathrm{p}<.05$, to the control group; the MPS and MPS by Group effects were not significant. These results indicate that practice in learning successive 


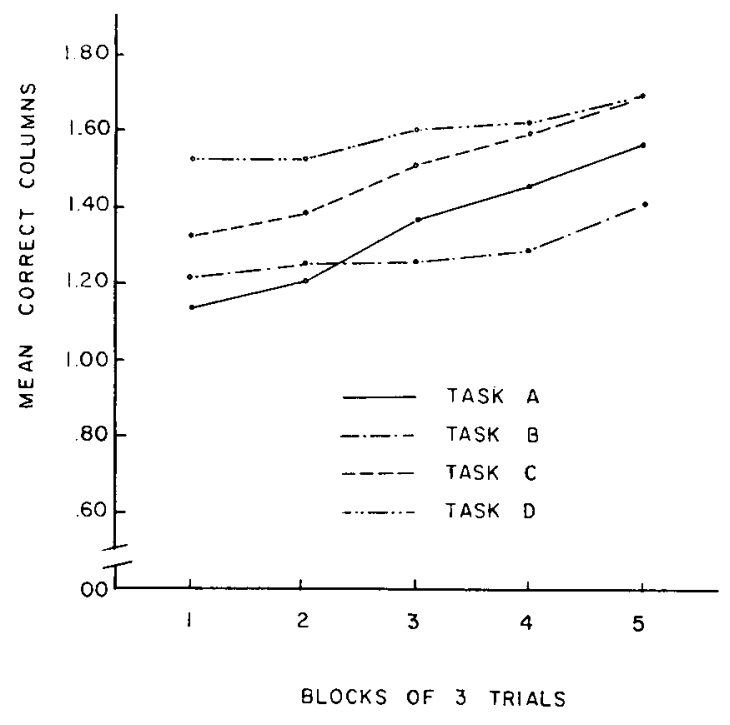

Fig. 1. Performance of transfer group in tasks A-D. Patterns representing different schemata were reproduced in each task.

schemata does lead to a general improvement in schema learning and that, in this task, at least, the MPS's do not differ significantly in difficulty.

The performance of the transfer group in tasks A-D is presented in Fig. 1. Figure 1 shows that performance on the first five trials of each task was superior to performance on the first five trials of the immediately preceding task, corroborating the conclusion that a general improvement in schema learning occurred over tasks.

The use of a specific encoding principle is indicated by the improvement within each task as Ss learned the specific characteristics of the schema associated with the patterns in that task. Tasks A, C, and D produced relatively rapid improvement, as compared to task $B$. This slow improvement in task B was anticipated on the grounds that the first shift from one schema to another should produce maximum interference from the previously learned schema. Thus, schema learning, as indicated by the rate of performance improvement, would be retarded. This low rate of improvement, which might be regarded as negative transfer, appears to have been limited to task B. On task D, there was clear evidence of positive transfer. These results thus supported the view that studies of transfer which are limited to a single transfer may not adequately describe the phenomenon.

It is also of interest to note that the initial level of performance on task $B$ is above that of task $A$. This difference is probably due to a general practice effect which was demonstrated previously (Edmonds \& Evans, 1966a). The rate of improvement was so much slower than on task A, however, that the order of superiority is reversed on trials 8-15. Do these two curves indicate negative or positive transfer? The first six trials suggest positive transfer, while the last nine trials suggest negative transfer. Clearly, either characterization is an oversimplification. Two different processes, general proactive effect and schema conflict are apparently operating; they have opposite effects; they have their maximum effects at different points on the curves.

These observations illustrate a matter of possible importance to other transfer studies: Curves of performance as a function of experience typically require more than one parameter to characterize them. There is no reason to assume that all parameters are similarly affected by pretraining conditions. Furthermore, mean performance over a set of trials does not represent any single parameter but reflects a combination of slope being directly related to the number of trials over which the average is taken. In other transfer experiments, too, a more detailed analysis of performance as a function of trials might be rewarding.

\section{References}

Edmonds, E. M., \& Evans, S. H. Schema learning without a prototype. Psychon. Sci., 1966a, 5, 247-248,

Edmonds, E. M., \& Evans, S. H. Prediction of schema learning by linear regression. Psychon. Sci., 1966b, 5, 457-458.

Evans, S. H. A model for perceptual category formation. Unpublished doctoral dissertation. Texas Christian University, 1964.

Evans, S. H., \& Edmonds, E. M. Schema differentiation as a function of training. Psychon. Sci., 1966, 5, 303-304.

Harlow, H. F. The formation of learning sets. Psychol. Rev., 1949, $56,51-56$.

Mueller, M. R., Edmonds, E. M., \& Evans, S. H. The quantification of schema theory and its implication for various perceptual tasks. Paper presented at Southwestern Psychological Association, April, 1966. 